\title{
REFINED LONG-TIME ASYMPTOTICS FOR SOME POLYMERIC FLUID FLOW MODELS*
}

\author{
A. ARNOLD ${ }^{\dagger}$, J.A. CARRILLO ${ }^{\ddagger}$, AND C. MANZINI ${ }^{\S}$
}

\begin{abstract}
We consider a polymeric fluid model, consisting of the incompressible Navier-Stokes equations coupled to a non-symmetric Fokker-Planck equation. First, the existence of steady states and the exponential convergence to them in relative entropy are proved for the linear Fokker-Planck equation in the Hookean case. The FENE model is also addressed, and the proof of the existence of stationary states and the convergence towards them in suitable weighted norms is given. Then, using the "entropy method" exponential convergence to the steady state is established for the coupled model in the Hookean case under some smallness assumption. The results continue and expand the analysis of [B. Jourdain, C. Le Bris, T. Lelièvre and F. Otto, Arch. Rational Mech. Anal., 181, $97-148,2006]$ in both the Hookean and the FENE models.
\end{abstract}

Key words. Entropy method, relative entropy, Fokker-Planck equations, large time behavior, exponential decay rate, polymeric flow, dumbbell model.

AMS subject classifications. 35K15, 35Q30, 35B40, 76T20.

\section{Introduction}

We consider a coupled microscopic-macroscopic model for a dilute solution of polymers in a homogeneous fluid. The incompressible Navier-Stokes equations for the macroscopic flow shall be coupled via the stress tensor to a microscopic model for the polymer chains distributed within the fluid (cf. [9, 10, 14, 28] for the physical background of such models). Let us briefly review the coupled model for the polymer distribution within a macroscopic flow. After putting the system in non-dimensional form and setting all remaining dimensionless parameters equal to one for notational simplicity, it reads as follows:

$$
\begin{aligned}
\frac{\partial u}{\partial t}(t, x)+\left(u(t, x) \cdot \nabla_{x}\right) u(t, x) & =\Delta_{x} u(t, x)-\nabla_{x} p(t, x)+\operatorname{div}_{x} \tau(t, x), \\
\operatorname{div}_{x} u & =0, \\
\tau(t, x) & =\int_{\mathbb{R}^{d}}\left(X \otimes \nabla_{X} \Pi(X)\right) \psi(t, x, X) d X,
\end{aligned}
$$

where $u(t, x), x \in \Omega \subset \mathbb{R}^{d}, d \geq 2$ is the velocity field of the fluid, $p(t, x)$ is the pressure, and $\tau(t, x)$ is the stress tensor. This system is coupled through equation (1.3) to the following microscopic model for the polymer evolution. Here, the polymers are modeled as dumbbells of length and orientation given by the vector $X \in \mathbb{R}^{d}$. The Fokker-Planck type evolution equation for $\psi(t, x, X)$, the probability density of dumbbells w.r.t. the (microscopic) extension $X$ at time $t$ and (macroscopic) position

\footnotetext{
*Recieved: April 17, 2009; Accepted (in revised form): October 4, 2009.

$\dagger$ Institute for Analysis and Scientific Computing, TU Wien, Wiedner Hauptstr. 8, A-1040 Wien, Austria (anton.arnold@tuwien.ac.at).

‡ICREA (Institució Catalana de Recerca i Estudis Avançats) and Departament de Matemàtiques, Universitat Autònoma de Barcelona, E-08193 Bellaterra, Spain (carrillo@mat.uab.es).

$\S$ Dept. of Applied Mathematics "G. Sansone", University of Florence, Via S. Marta 3, I-50139 Firenze, Italy (manzinimeister@gmail.com).
} 
$x$ reads

$$
\begin{aligned}
\frac{\partial \psi}{\partial t}(t, x, X) & +\left(u(t, x) \cdot \nabla_{x}\right) \psi(t, x, X) \\
= & -\operatorname{div}_{X}\left(\left[\left(\nabla_{x} \otimes u(t, x)\right)^{T} \cdot X-\frac{1}{2} \nabla_{X} \Pi(X)\right] \psi(t, x, X)\right) \\
& +\frac{1}{2} \Delta_{X} \psi(t, x, X) .
\end{aligned}
$$

Here, $\nabla_{X} \Pi(X)$ denotes the recovering force field between the two beads of the dumbbells modelled as a spring with potential $\Pi(X)$. The term $\left(\nabla_{x} \otimes u(t, x)\right)^{T} \cdot X$ comes from the deformation of the dumbbell extensions due to the stress forces produced by the inhomogeneous flow field $u$. Actually, the derivatives in the Fokker-Planck equation involving the velocity $u$ can be considered as the Eulerian terms coming from a microscopic Lagrangian description; see [9, 28, 15, 18] for a full discussion of the model. The model of Hookean dumbbells is obtained by setting the elastic spring potential $\Pi$ as $\Pi(X)=|X|^{2}$, while the finite extensible nonlinear elasticity (FENE) model of polymeric fluids comes from choosing

$$
\Pi(X)=-\frac{b}{2} \ln \left(1-\frac{|X|^{2}}{b}\right)
$$

with $b \geq 2$. Particularly the FENE-model has attracted lots of mathematical interest in the last few years $[33,27,24,12]$ (with some of that work having been carried out in parallel to this paper). The singularity of $\Pi$ at $|X|=\sqrt{b}$ makes (1.4) degenerate (both in the parabolic and elliptic case) and hence quite challenging.

Under suitable boundary conditions, equations (1.1)-(1.4) admit special solutions in the form of homogeneous flows (i.e., $\left.\left(\nabla_{x} \otimes u\right)^{T}=\kappa\right)$ with a stationary distribution of the polymer extensions. The stability of such solutions will be one topic of this paper. But first we shall focus on the $x$-homogeneous equation associated to (1.4) and the coupled system (1.1)-(1.4). Using the entropy-entropy dissipation method we shall analyze its large-time behavior. In particular we prove its exponential convergence to equilibrium solutions in the form of homogeneous stationary flows.

The goal of this paper is to answer several open questions posed in [19], more precisely:

A. Hookean case: existence and uniqueness of stationary states for a general deformation matrix $\kappa$ and convergence in relative entropy to them, both for the $x$-homogenous case and in the coupled case under the assumption of a small deformation matrix.

B. FENE case: existence, uniqueness, and asymptotic stability for the $x$ homogenous case in weighted $L^{2}$-spaces in a more general setting than in [19].

But we cannot yet conclude large time convergence in relative entropy in the FENE case, as we are still lacking certain bounds on the stationary states, see section 2.4. This relative entropy convergence result would immediately imply asymptotic stability results of these homogeneous stationary flows for the coupled system for the FENE case by repeating the arguments of [19, Theorem 1] or [19, Proposition 9].

The paper is organized as follows. In section 2 we study the linear Fokker-Planck equation (1.4) for the dumbbell distribution in a given, homogeneous flow field $\nabla_{x} \otimes$ $u=\kappa$. Stationary states $\psi_{\infty}$ and exponential convergence of $\psi(t, X)$ towards them are 
established for Hookean dumbbells (for all matrices $\kappa$ such that $2 \kappa-I$ is stable) and the FENE model (under some smallness conditions on $\kappa$ ). In section 3 we analyze the coupled system in the Hookean case with non-homogeneous Dirichlet boundary conditions on $u$. Under a smallness assumption on $\kappa$ and $X$-moments on $\psi$, we prove exponential convergence of $(u(t), \psi(t))$ to the homogeneous stationary flow $\left(u_{\infty}, \psi_{\infty}\right)$.

\section{Homogeneous flow with a given velocity field}

In this section we consider the case that the velocity field $u$ is given and that there exists an arbitrary (but constant in $t$ and $x$ ) matrix $\kappa \in \mathbb{R}^{d \times d}$ such that

$$
u(t, x)=\kappa x .
$$

Then we rewrite equation (1.4) for the unknown $\psi=\psi(t, X)$, which is now independent of the space variable $x$ :

$$
\begin{aligned}
\frac{\partial \psi}{\partial t}(t, X) & =L \psi(t, X), \quad t>0, \quad X \in \mathbb{R}^{d}, \\
L \psi(X) & :=\frac{1}{2} \operatorname{div}_{X}\left(\left[\nabla_{X} \Pi(X)-2 \kappa X\right] \psi(X)\right)+\frac{1}{2} \Delta_{X} \psi(X), \\
\psi(0, X) & =\psi_{0}(X) .
\end{aligned}
$$

Assuming $\psi_{0} \geq 0$ implies that $\psi(t, X) \geq 0$ for $t>0$ by a parabolic maximum principle. Moreover, the divergence form of (2.2) implies that $\psi$ stays normalized under time evolution: $\int \psi(t, X) d X=\int \psi_{0}(X) d X=1$.

Now we shall analyze the large-time behavior of (2.2) for three types of (given) potentials $\Pi(X)$ : Hookean dumbbells, section 2.2, the finite extensible nonlinear elasticity (FENE) model of polymeric fluids, section 2.4, and general (radially symmetric) potentials in the special case that $\kappa$ is a normal matrix, section 2.3. To this end we shall apply the entropy-entropy dissipation method (cf. $[4,6,26,2]$, e.g.).

2.1. Entropy-entropy dissipation method. Let us briefly summarize for later reference the main definitions and the steps of the entropy-entropy dissipation method. It aims at deriving estimates for the relative entropy of the solution $\psi(t)$ w.r.t. the steady state $\psi_{\infty}$. For $\psi, \varphi$, two probability densities on $\mathbb{R}^{d}$, the (logarithmic) relative entropy is defined as

$$
e(\psi \mid \varphi):=\int_{\mathbb{R}^{d}} \psi(X) \ln \frac{\psi(X)}{\varphi(X)} d X \geq 0 .
$$

Since it satisfies the Csiszár-Kullback inequality [13, 21]

$$
\|\psi-\varphi\|_{L^{1}\left(\mathbb{R}^{d}\right)}^{2} \leq 2 e(\psi \mid \varphi),
$$

it is a measure for the "distance" of $\psi$ to $\varphi$.

To apply the entropy-entropy dissipation method for non-symmetric FokkerPlanck equations (cf. section 2.4 of [4]) we shall proceed in three steps: Firstly, we shall prove the existence of a unique normalized steady state of (2.2). In several cases it is possible to derive an explicit formula for $\psi_{\infty}$ (cf. sections 2.2, 2.3).

In the second step we use the unique normalized steady state $\psi_{\infty}$ to split the drift vector field in (2.2) as

$$
\nabla \Pi(X)-2 \kappa X=\nabla A(X)+\vec{F}(X),
$$


with

$$
A(X):=-\ln \left(\psi_{\infty}(X)\right) .
$$

Since $\psi_{\infty}$ is a steady state of $(2.2)$, we obtain from

$$
\operatorname{div}_{X}\left(\nabla_{X} A(X) \psi_{\infty}+\nabla_{X} \psi_{\infty}\right)=0
$$

that the (non-gradient) vector field $\vec{F}$ satisfies

$$
\operatorname{div}_{X}\left(\vec{F} \psi_{\infty}\right)=0 .
$$

Accordingly, finding the steady state $\psi_{\infty}$ of (2.2) is equivalent to decomposing the given vector field (2.4) into a gradient field $(\nabla A)$ and a divergence-free field $(\vec{F}$, in the sense of (2.7)). This resembles the Helmholtz-Hodge decomposition in incompressible fluid mechanics, and we shall illustrate this analogy in subsequent examples. Another consequence of the above splitting of the drift field is the decomposition of the generator $L$ into its symmetric and anti-symmetric parts in $\mathcal{X}:=L^{2}\left(\psi_{\infty}^{-1} d X\right)$ :

$$
\begin{aligned}
L^{\mathrm{s}} \psi & :=\frac{1}{2} \operatorname{div}_{X}\left(\nabla A(X) \psi+\nabla_{X} \psi\right)=\frac{1}{2} \operatorname{div}_{X}\left(\psi_{\infty} \nabla_{X} \frac{\psi}{\psi_{\infty}}\right), \\
L^{\mathrm{as}} \psi & :=\frac{1}{2} \operatorname{div}_{X}(\vec{F}(X) \psi) .
\end{aligned}
$$

Note that $L^{s} \leq 0$, since we have (somewhat formally, as $\mathcal{D}\left(L^{s}\right)$ is not yet defined):

$$
\left\langle L^{s} \psi, \psi\right\rangle_{\mathcal{X}}=\frac{1}{2} \int_{\mathbb{R}^{d}} \operatorname{div}_{X}\left(\psi_{\infty} \nabla_{X} \frac{\psi}{\psi_{\infty}}\right) \frac{\psi}{\psi_{\infty}} d X=-\frac{1}{2} \int_{\mathbb{R}^{d}}\left|\nabla_{X} \frac{\psi}{\psi_{\infty}}\right|^{2} \psi_{\infty} d X \leq 0 .
$$

Moreover, $\psi_{\infty}$ is not only the steady state of the non-symmetric Fokker-Planck equation (2.2) but also of its "symmetric part" $\psi_{t}=L^{s} \psi$ (since $L^{s} \psi_{\infty}=0$ ).

The third step consists in applying Theorem 2.19 of [4]: The entropy decay of solutions to the non-symmetric Fokker-Planck equation (2.2) is at least as fast as the decay rate for the corresponding symmetric Fokker-Planck equation $\psi_{t}=L^{s} \psi$.

2.2. Hookean dumbbells. Here we assume that

$$
\Pi(X)=\frac{|X|^{2}}{2} .
$$

The resulting model (2.2), (2.10) was already analyzed in section 2.1 of [19]. There the authors established a unique (normalized) steady state $\psi_{\infty}$ and the exponential decay of the relative entropy for the two cases: $\kappa$ either symmetric or antisymmetric. But the generic case was left as an open problem. The following results close this gap in a unified approach. The next proposition constitutes the first step of the entropy-entropy method:

Proposition 2.1. Let the potential $\Pi$ be defined by (2.10) and let the eigenvalues of the matrix $\kappa$ satisfy $\Re \lambda_{j}(\kappa)<\frac{1}{2}, j=1, \ldots, d$. Then there exists a unique normalized steady state $\psi_{\infty} \in L^{1}\left(\mathbb{R}^{d}\right) \cap H^{2}\left(\mathbb{R}^{d}\right) \cap H^{1}\left(\mathbb{R}^{d} ;|X| d X\right)$ for equation (2.2). It has the form

$$
\psi_{\infty}(X)=(2 \pi)^{-d / 2}(\operatorname{det} \Sigma)^{-1 / 2} \exp \left(-\frac{1}{2} X^{T} \Sigma^{-1} X\right)
$$


with the symmetric, positive definite matrix $\Sigma$ given by

$$
\Sigma=2 \int_{0}^{\infty} e^{-(I-2 \kappa) \theta} e^{-\left(I-2 \kappa^{T}\right) \theta} d \theta
$$

Here, $\kappa^{T}$ denotes the transpose of $\kappa$ and I the identity matrix. Moreover, the spectral condition that the eigenvalues of the matrix $\kappa$ satisfy $\Re \lambda_{j}(\kappa)<\frac{1}{2}, j=1, \ldots, d$ is necessary for the existence of a stationary normalized solution of the form (2.11).

REMARK 2.2. For $\kappa$ normal, (2.12) simplifies to

$$
\Sigma=\left(I-2 \kappa^{s}\right)^{-1}
$$

with $\kappa^{s}:=\left(\kappa+\kappa^{T}\right) / 2$.

Proposition 2.1 makes use of the following lemma (cf. [11, 31], section 2.2 of [17]). LEMma 2.3. Consider the continuous Lyapunov equation

$$
B \Sigma+\Sigma B^{H}+Q=0
$$

for the $d \times d$ matrix $\Sigma$ with a given Hermitian and positive definite $d \times d$ matrix $Q$. A necessary and sufficient condition for the existence of a positive definite, Hermitian solution is that the $d \times d$ matrix $B$ is stable (i.e. $\left.\Re \lambda_{j}(B)<0, j=1, \ldots, d\right)$. Then, the unique solution is given by

$$
\Sigma=\int_{0}^{\infty} e^{B \theta} Q e^{B^{H} \theta} d \theta
$$

The solution of (2.14) can be computed by a standard numerical algorithm [8], which is also implemented in $M A T L A B$, e.g.

Proof of Proposition 2.1.

Proof. The stationary version of equation (2.2) with Hookean potential (2.10) reads

$$
-\operatorname{div}_{X}((2 \kappa-I) X \psi(X))+\Delta_{X} \psi(X)=0 .
$$

From this it is natural to assume that $2 \kappa-I$ is a stable matrix for a confinement on $\psi$ to exist. Next we Fourier transform $(2.16)$, denoting $\widehat{\psi}(\xi):=\mathcal{F}_{X \rightarrow \xi} \psi(X)$ :

$$
\xi^{T}(I-2 \kappa) \nabla_{\xi} \widehat{\psi}(\xi)=-|\xi|^{2} \widehat{\psi}(\xi) .
$$

The normalization then reads

$$
\widehat{\psi}(0)=(2 \pi)^{-d / 2} \int_{\mathrm{IR}^{d}} \psi(X) d X=(2 \pi)^{-d / 2} .
$$

We consider the characteristic system for (2.17),

$$
\dot{\xi}=\xi^{T}(I-2 \kappa) \text {. }
$$

Since $2 \kappa-I$ is stable, all characteristic curves are spirals that connect $\xi=0$ to $\infty$. Since we seek a solution $\psi \in L^{1}\left(\mathbb{R}^{d}\right)$, we have $\widehat{\psi} \in C\left(\mathbb{R}^{d}\right)$. Using (2.18) as an initial condition for each characteristic, we find that $(2.17),(2.18)$ has a unique global 
solution on all of $\mathbb{I R}^{d}$. (2.17) shows that $\widehat{\psi}$ decays to 0 along each characteristic as $X \rightarrow \infty$.

Actually, $\widehat{\psi}$ can be computed explicitly. Using the ansatz

$$
\widehat{\psi}(\xi)=\frac{1}{Z} \exp \left(-\frac{1}{2} \xi^{T} \Sigma \xi\right)
$$

with a positive definite, symmetric matrix $\Sigma$ and normalization constant $Z$, equation (2.17) reduces to

$$
\xi^{T}((I-2 \kappa) \Sigma-I) \xi=0, \forall \xi \in \mathbb{R}^{d}
$$

which is equivalent to $0=-2((I-2 \kappa) \Sigma-I)^{s}=-(I-2 \kappa) \Sigma-\Sigma(I-2 \kappa)^{T}+2 I$. This is a continuous Lyapunov equation for $\Sigma$. Then, Lemma 2.3 guarantees the existence of a unique positive definite and symmetric matrix $\Sigma$, since $2 \kappa-I$ is stable and $2 I$ is positive definite and symmetric. Inverse Fourier transformation and normalization yields (2.11). (2.13) is readily obtained by diagonalizing the normal matrix $\kappa$.

The fact that the spectral condition is necessary is included in Lemma 2.3.

An expression closely related to (2.12) is given in [19], Remark 10 for the stationary stress tensor $\tau_{\infty}(x)$ in a homogeneous stationary flow.

So far we have established the existence of a unique normalized steady state of Gaussian shape $\psi_{\infty}(X)=\exp (-A(X))$ where $A(X)$ is a quadratic polynomial (cf. (2.11)). In order to prove exponential convergence of $\psi(t)$ to the steady state $\psi_{\infty}$, we apply the entropy-entropy dissipation method for "non-symmetric diffusion equations" as outlined in section 2 .

First we rewrite $(2.2),(2.10)$ in the following "split form":

$$
\frac{\partial \psi}{\partial t}(t, X)=\frac{1}{2} \operatorname{div}_{X}\left(\left(\nabla_{X} A(X)+\vec{F}(X)\right) \psi(t, X)\right)+\frac{1}{2} \Delta_{X} \psi(t, X),
$$

with $A(X):=-\ln \left(\psi_{\infty}(X)\right)=\frac{1}{2} X^{T} \Sigma^{-1} X+$ const and

$$
\vec{F}(X)=(I-2 \kappa) X-\nabla_{X} A(X)=\left(I-2 \kappa-\Sigma^{-1}\right) X .
$$

COROLlary 2.4. Under the assumptions of Proposition 2.1, equations (2.2), (2.10) can be written in the "split-form" (2.20). In addition to (2.7), the (non-gradient) vector field $\vec{F}$ defined by (2.21) also satisfies

$$
\operatorname{div}_{X}(\vec{F})=0 .
$$

As a consequence the splitting in (2.20) provides the "pointwise" Helmholtz-Hodge decomposition of the vector field

$$
\nabla \Pi-2 \kappa X=\nabla A+\vec{F} .
$$

Proof. Equation (2.22) follows from

$$
0=\operatorname{div}_{X}\left[\vec{F} \mathrm{e}^{-A(X)}\right]=\mathrm{e}^{-A(X)}\left(\operatorname{div}_{X} \vec{F}-\nabla_{X} A \cdot \vec{F}\right),
$$

where

$$
\nabla_{X} A \cdot \vec{F}=X^{T} \Sigma^{-1}\left(I-2 \kappa-\Sigma^{-1}\right) X=\left(X^{T} \Sigma^{-1}\right)[(I-2 \kappa) \Sigma-I]\left(\Sigma^{-1} X\right)=0
$$


by $(2.19)$.

Using (2.21), equation (2.22) implies $\operatorname{Tr}\left(I-2 \kappa-\Sigma^{-1}\right)=0$, and hence $\operatorname{Tr} \Sigma^{-1} \in \mathbb{R}$ is always explicitly computable:

$$
\operatorname{Tr} \Sigma^{-1}=\operatorname{Tr}\left(I-2 \kappa^{s}\right)=d-2 \sum_{j=1}^{d} \Re \lambda_{j}(\kappa)>0 .
$$

Compare this expression to (2.13), which holds in the special case $\kappa$ normal (Remark $2.2)$.

By using the third step of the entropy-entropy method in section 2.1, we can prove the following.

TheOREM 2.5. Let $\psi_{0} \in L^{1}\left(\mathbb{R}^{d}\right)$ be a probability density with $e\left(\psi_{0} \mid \psi_{\infty}\right)<\infty$. Under the assumptions of Proposition 2.1, we have exponential convergence of $\psi(t)$ towards $\psi_{\infty}$ in relative entropy with rate $\lambda_{\min }\left(\Sigma^{-1}\right)>0$ :

$$
e\left(\psi(t) \mid \psi_{\infty}\right) \leq e^{-\lambda_{\min }\left(\Sigma^{-1}\right) t} e\left(\psi_{0} \mid \psi_{\infty}\right), \quad t \geq 0 .
$$

Proof. The decomposition of the generator $L$ (cf. (2.8)) simplifies in the Hookean case to

$$
L^{s} \psi=\frac{1}{2} \operatorname{div}\left(\Sigma^{-1} X \psi+\nabla \psi\right), \quad L^{a s} \psi=\frac{1}{2} \vec{F} \cdot \nabla \psi .
$$

Section 2.4 of [4] now applies directly to the non-symmetric Fokker-Planck equation (2.20). The entropy decay of its solution is at least as fast as that of the symmetric counterpart $\psi_{t}=L^{s} \psi$. Using the Bakry-Emery convexity condition (cf. $[6,7,4]$ ) for $A(X)$, the decay rate is given by $\lambda_{\min }(\operatorname{Hess}(A))=\lambda_{\min }\left(\Sigma^{-1}\right)>0$, the minimal eigenvalue of the matrix $\Sigma^{-1}$. Hence we have proved the thesis.

In the two special cases of $\kappa$ either symmetric or antisymmetric we recover the results of [19]. For $\kappa$ symmetric, we have $\Sigma=(I-2 \kappa)^{-1}$ and the decay rate of $e(t)$ is $1-2 \lambda_{\max }(\kappa)>0$, just as in Proposition 1(iv) of [19]. For $\kappa$ antisymmetric, we obtain $\Sigma=I, \psi_{\infty}(X)=(2 \pi)^{-d / 2} e^{-\Pi(X)}$, and the decay rate is 1 (like in Proposition $1(i)$ of $[19])$.

REMARK 2.6. The entropy decay rate of Theorem 2.5 is actually sharp which can be seen as follows. "Optimal functions" for the entropy decay of the symmetric FokkerPlanck equation $\psi_{t}=L^{s} \psi$ (with the quadratic potential $\frac{1}{2} X^{T} \Sigma^{-1} X$ ) are the shifted Maxwellians $\mu(X):=\rho_{\infty}\left(X-\xi e_{1}\right), \xi \in \mathbb{R} \backslash\{0\}$, where $e_{1}$ is an eigenvector of $\Sigma^{-1}$ for $\lambda_{\min }\left(\Sigma^{-1}\right)$ (cf. section 3.5 of [4]). This means that the entropy decay for the symmetric Fokker-Planck equation with $\psi_{0}=\mu$ is exactly exponential with the rate $\lambda_{\min }\left(\Sigma^{-1}\right)$. Note that $\mu$ is also an "optimal function" of the corresponding Logarithmic Sobolev inequality which makes it an equality.

Now we recall that, for a non-symmetric Fokker-Planck equation, the relative entropy $e\left(\psi_{0} \mid \psi_{\infty}\right)$ and the entropy dissipation

$$
\left.\frac{d}{d t} e\left(\psi(t) \mid \psi_{\infty}\right)\right|_{t=0}=-\frac{1}{2} \int_{\mathbb{R}^{d}}\left|\nabla \frac{\psi_{0}}{\psi_{\infty}}\right|^{2} \frac{\psi_{\infty}^{2}}{\psi_{0}} d X
$$


both coincide with the terms in its symmetric counterpart - the entropy by definition and the entropy dissipation because of

$$
\int\left(L^{a s} \psi_{0}\right) \ln \frac{\psi_{0}}{\psi_{\infty}} d X=-\frac{1}{2} \int \psi_{\infty} \vec{F} \cdot \nabla \frac{\psi_{0}}{\psi_{\infty}} d X=\frac{1}{2} \int \operatorname{div}\left(\vec{F} \psi_{\infty}\right) \frac{\psi_{0}}{\psi_{\infty}} d X=0
$$

(cf. section 2.4 of [4]). Hence, for $\psi_{0}=\mu$ and $t=0$ the time-derivative of both sides in (2.23) coincide, which rules out any better decay rate in Theorem 2.5.

REMARK 2.7. In $[2,1]$ an alternative entropy method for non-symmetric diffusion equations was developed. There, the exponential decay rate of $e(t)$ is estimated by the uniform convexity of

$$
\frac{\partial^{2} A}{\partial X^{2}}-\frac{1}{2}\left(\frac{\partial \vec{F}}{\partial X}+\left(\frac{\partial \vec{F}}{\partial X}\right)^{T}\right)
$$

In the Hookean case this lower convexity bound is $\tilde{\lambda}:=\lambda_{\min }\left(2 \Sigma^{-1}-I+2 \kappa^{s}\right)$ with $\Sigma=\Sigma(\kappa)$ given by (2.12). As discussed in Remark 2.6 it has to satisfy $\tilde{\lambda} \leq \lambda_{\min }\left(\Sigma^{-1}\right)$ (which is easily verified numerically). Hence, this approach does not yield a "better" decay rate for the Hookean dumbbell model having a homogeneous vector field $\vec{F}(X)=\left(I-2 \kappa-\Sigma^{-1}\right) X$. This was to be expected from the examples given in [2], where improved decay rates were obtained only from highly non-homogeneous vector fields $\vec{F}(X)$.

2.3. General potential with a normal matrix $\kappa$. Here we consider $(2.2)$ with a radially symmetric potential $\Pi(X)=\pi(|X|)$. While we present here the whole space problem $X \in \mathbb{R}^{d}$, the same argument applies to bounded domain models as is section 2.4. For $\kappa$ normal we have the following generalization of Proposition 2.1 (cf. $(2.13))$.

Proposition 2.8. Let $\kappa$ be a normal matrix, and let the potential $\Pi$ and $\kappa$ satisfy $\exp \left[-\Pi(X)+X^{T} \kappa^{s} X\right] \in L^{1}\left(\mathbb{R}^{d}\right)$. Then

$$
\psi_{\infty}(X)=C e^{-\Pi(X)+X^{T} \kappa^{s} X},
$$

with some appropriate constant $C$, is a normalized steady state of (2.2).

Proof. Using the matrix decomposition $\kappa=\kappa^{\mathrm{s}}+\kappa^{\text {as }}$, a straightforward computation yields for all $X \in \mathbb{R}^{d}$ :

$$
L \psi_{\infty}=-\operatorname{div}\left(\kappa^{\mathrm{as}} X \psi_{\infty}\right)=X^{T} \kappa^{\mathrm{as}}\left[2 \kappa^{\mathrm{s}} X-\frac{X}{|X|} \pi^{\prime}(|X|)\right] \psi_{\infty}=\frac{1}{2} X^{T}\left[\kappa, \kappa^{T}\right] X \psi_{\infty}=0 .
$$

We remark that for the FENE model, this form of the steady state could also have been deduced from the estimate (102) in [19].

Now we proceed as in section 2.2 and define

$$
A(X):=-\ln \left(\psi_{\infty}(X)\right)=\Pi(X)-X^{T} \kappa^{s} X+\text { const }, \quad \vec{F}(X)=-2 \kappa^{\text {as }} X
$$

as the coefficients of the Fokker-Planck equation in "split form" (2.20). It satisfies $\operatorname{div} \vec{F}=0, \vec{F} \cdot \nabla A=0$. The entropy-entropy dissipation method then yields the following theorem. 
Theorem 2.9. Let $\psi_{0} \in L^{1}\left(\mathbb{R}^{d}\right)$ be a probability density with $e\left(\psi_{0} \mid \psi_{\infty}\right)<\infty$ and assume that $\lambda:=\inf _{X \in \mathbb{R}^{d}}\left[\lambda_{\min }\left(\operatorname{Hess}(\Pi(X))-2 \kappa^{s}\right)\right]>0$. Under the assumptions of Proposition 2.8, we have exponential convergence of $\psi(t)$ towards $\psi_{\infty}$ in relative entropy with rate $\lambda$ :

$$
e\left(\psi(t) \mid \psi_{\infty}\right) \leq e^{-\lambda t} e\left(\psi_{0} \mid \psi_{\infty}\right), \quad t \geq 0
$$

We remark that an alternative decay rate can be obtained by considering $A$ as an $L^{\infty}$-perturbation of the uniformly convex potential $\Pi$ and applying a Holley-Stroock perturbation argument for logarithmic Sobolev inequalities (cf. [16, 4]). Particularly for bounded domain models (like the FENE model of section 2.4) this may yield a better decay rate.

Corollary 2.10. Under the assumptions of Theorem 2.9, $\psi_{\infty}$ from (2.24) is the unique normalized steady state of (2.2) within the set $\left\{\psi \in L_{+}^{1}\left(\mathbb{R}^{d}\right) \mid \int_{\mathbb{R}^{d}} \psi d X=\right.$ $\left.1, e\left(\psi \mid \psi_{\infty}\right)<\infty\right\} \supset\left\{\psi \in L_{+}^{2}\left(\mathbb{R}^{d} ; \psi_{\infty}^{-1} d X\right) \mid \int_{\mathbb{R}^{d}} \psi d X=1\right\} \quad(c f$. [4]).

Let us finally mention a weaker condition for uniqueness of the steady state. Let the coefficient $A(X)$ be such that the operator $L^{s}$ has a positive spectral gap when considered on $L^{2}\left(\psi^{-1} d X\right)$. This would then imply exponential convergence of $\psi(t)$ towards $\psi_{\infty}$ in the $L^{2}\left(\psi^{-1} d X\right)$-norm (cf. [4] for details).

2.4. FENE potential. Here, we will improve on the hypotheses for the existence, uniqueness, and stability of stationary states compared to section 2.1 of [19]. More precisely, the results in [19] show that when $\kappa$ is a general traceless matrix with $\kappa^{s}$ "small" (i.e. $\left|\kappa^{s}\right|<1 / 2$ ), then stationary states exist and asymptotic stability is obtained by a Holley-Stroock perturbation argument. We will show that the existence and uniqueness of stationary states and their asymptotic stability can be established from a pure linear operator theory point of view in weighted Sobolev spaces. This leads to the answer to these questions under less restrictive hypotheses than in [19]. However, we do not know in general how to prove convergence in relative entropy due to the lack of pointwise control of the behavior close to the boundary of the stationary states. Now, we consider equation (2.2)

$$
\frac{\partial \psi}{\partial t}=\frac{1}{2} \operatorname{div}_{X}\left(\left[\nabla_{X} \Pi(X)-2 \kappa X\right] \psi\right)+\frac{1}{2} \Delta_{X} \psi,
$$

with the potential $\Pi$ given by

$$
\Pi(X)=-\frac{b}{2} \ln \left(1-\frac{|X|^{2}}{b}\right),
$$

for some $b \geq 2$. For a discussion of the physical relevance of this lower bound we refer to [10] and section 1.1 of [19]. Also mathematically, $b=2$ is a critical value for the boundary behaviour of solutions (cf. [24] and Lemma 2.11 below). In the FENE-model the polymer chains are assumed to have finite extensibility. This is reflected by $\Pi(X) \rightarrow+\infty$ for $|X|^{2} \rightarrow b$. Hence, it is natural to study the problem in the ball of radius $\sqrt{b}, \mathcal{B}=\mathcal{B}(0, \sqrt{b})$ with a no-flux boundary condition on $\partial \mathcal{B}$. Then $\psi$ also satisfies a homogeneous Dirichlet boundary condition (cf. section 1.1 of [19] for details). Due to the singularity of $\Pi$ at $\partial \mathcal{B}$, it is actually not even necessary to prescribe any boundary data for the parabolic initial value problem $(2.25)-(2.26)$. For $\psi$ smooth enough, it will "automatically" have a zero trace on $\partial \mathcal{B}$ (cf. $[33,24,25]$ ). 
Our first goal (cf. section 2.1) is to prove that the degenerate elliptic equation

$$
\begin{aligned}
L \psi(X):=\frac{1}{2} \operatorname{div}_{X}\left(\left[\nabla_{X} \Pi(X)-2 \kappa X\right] \psi(X)\right)+\frac{1}{2} \Delta_{X} \psi(X) & =0, & \mathcal{B}, \\
\psi & =0, & \partial \mathcal{B}
\end{aligned}
$$

has a unique normalized solution. In contrast to section 2.2 , section 2.3 here we do not know the explicit form of the steady state (at least for $\kappa$ not normal). This prevents us from using for $(2.25),(2.26)$, at least at the very beginning, the canonical decomposition (2.4), (2.5) of its drift field. As an alternative, we shall instead use a perturbation argument to establish the existence of a steady state. In particular we shall discuss the following four cases, where $\kappa$ is either a normal matrix or "close" to a normal matrix:

(i) $\kappa$ is symmetric (see Example 2.15 below) or anti-symmetric (see Example 2.16);

(ii) the commutator $\left[\kappa, \kappa^{T}\right]$ is small (see Example 2.15), or $\kappa$ has a small distance to the set of normal matrices (see Example 2.17).

We first use an auxiliary decomposition of $\kappa$ as $\kappa=\kappa_{1}+\kappa_{2}$, with $\kappa_{1}$ a normal matrix to be chosen later. Inspired by the steady state function in the case $\kappa$ normal (cf. (2.24)) we set

$$
\mu(X):=C e^{-\Pi(X)+X^{T} \kappa_{1}^{s} X}=C\left(1-\frac{|X|^{2}}{b}\right)^{\frac{b}{2}} e^{X^{T} \kappa_{1}^{s} X},
$$

with $\kappa_{1}^{\mathrm{s}}:=\left(\kappa_{1}+\kappa_{1}^{T}\right) / 2$ and the normalization $\int_{\mathcal{B}} \mu d X=1$. Clearly, $\mu=0$ on $\partial \mathcal{B}$, and for $b>2$ it also takes homogeneous Neumann boundary values.

In analogy to [5], [27] we define the following weighted $H_{0}^{1}$-space:

$$
\mathcal{V}:=\left\{\psi \mid \frac{\psi}{\mu}, \nabla \frac{\psi}{\mu} \in L^{2}(\mathcal{B} ; \mu d X)\right\},
$$

i.e. $\frac{\psi}{\mu} \in H^{1}(\mathcal{B} ; \mu d X)=H_{0}^{1}(\mathcal{B} ; \mu d X)$ (cf. Proposition 9.10 of $\left.[20]\right) . \mathcal{V}$ is endowed with the obvious norm

$$
\|\psi\|_{\mathcal{V}}^{2}=\left\|\frac{\psi}{\mu}\right\|_{L^{2}(\mathcal{B} ; \mu d X)}^{2}+\left\|\nabla \frac{\psi}{\mu}\right\|_{L^{2}(\mathcal{B} ; \mu d X)}^{2} .
$$

$\mathcal{V}$ is a separable Hilbert space and a dense subset of $\mathcal{H}:=L^{2}\left(\mathcal{B} ; \mu^{-1} d X\right.$ ) (cf. [32], section 3.2.2). We shall denote the $\mathcal{H}$-inner product by $\langle\cdot, \cdot\rangle$. Note that the space $\mathcal{V}$ is independent of the decomposition $\kappa=\kappa_{1}+\kappa_{2}$ (with equivalent norms), which is seen as follows. Since $\frac{1}{\alpha} \leq e^{X^{T} \kappa_{1}^{\mathrm{s}} X} \leq \alpha$ on $\mathcal{B}$ for some $\alpha>0$, this independence is trivial concerning $\frac{\psi}{\mu} \in L^{2}(\mathcal{B} ; \mu d X)$. For the second term in the definition of $\mathcal{V}$ we use

$$
\nabla \frac{\psi}{\mu}=\frac{\nabla \psi+\psi \nabla \Pi}{\mu}-\frac{\psi}{\mu} 2 \kappa_{1}^{\mathrm{s}} X
$$

Hence, the second term in $(2.29)$ is always in $L^{2}(\mathcal{B} ; \mu d X)$, and for the first term we conclude as before.

Before deriving a weak formulation of $(2.27)$ in $\mathcal{V}$, it is in order to discuss the boundary traces of functions $\psi \in \mathcal{V}$. Similar to the parabolic case (2.25), we shall find that functions in $\mathcal{V}$ have a zero trace. Hence, we will actually not have to prescribe 
homogeneous Dirichlet data in (2.27). They are already implied by the functional setting, due to the singular weight in $\mathcal{V}$.

By definition we have $\frac{\psi}{\mu} \in H^{1}(\mathcal{B} ; \mu d X)$. In this weighted Sobolev space the weight function $\mu(X)$ behaves close to $\partial \mathcal{B}$ like the power function $d(X)^{b / 2}$, where $d(X):=$ $\sqrt{b}-|X|$ denotes the distance of $X$ from the boundary $\partial \mathcal{B}$. Hence, $H^{1}(\mathcal{B} ; \mu d X)=$ $H_{0}^{1}(\mathcal{B} ; \mu d X) \forall b \geq 2$ (cf. Proposition 9.10 of [20] or section 3.2 .2 of [32]), but $\frac{\psi}{\mu}$ does not necessarily have a (vanishing) trace (cf. Remark 9.17 of [20]). To proceed we now give a different characterization of $\mathcal{V}$.

LEMMA 2.11.

(a) Let $b>2$. Then

$$
\mathcal{V}=H^{1}\left(\mathcal{B} ; \mu^{-1} d X\right)=H_{0}^{1}\left(\mathcal{B} ; \mu^{-1} d X\right)
$$

and hence $\psi \in \mathcal{V}$ has a trace with $\left.\psi\right|_{\partial \mathcal{B}}=0$ a.e. (cf. Remark 9.17 of [20]). More precisely, there exists a (trivial) trace operator on $H^{1}\left(\mathcal{B} ; \mu^{-1} d X\right)$ which satisfies $\Gamma \psi=0$ (pointwise on $\partial \mathcal{B}) \forall \psi \in H^{1}\left(\mathcal{B} ; \mu^{-1} d X\right) \cap C^{\infty}(\overline{\mathcal{B}})$.

(b) Let $b=2$. Then

$$
\mathcal{V} \supset H^{1}\left(\mathcal{B} ; \mu^{-1} d X\right)=H_{0}^{1}\left(\mathcal{B} ; \mu^{-1} d X\right)
$$

Moreover, there exists a (trivial) trace operator $\Gamma: \mathcal{V} \rightarrow L^{2}(\partial \mathcal{B})$ which satisfies $\left.\psi\right|_{\partial \mathcal{B}}=0 \forall \psi \in \mathcal{V} \cap C^{\infty}(\overline{\mathcal{B}})$.

Proof. $\quad$ First we recall the identity $H^{1}\left(\mathcal{B} ; \mu^{-1} d X\right)=H_{0}^{1}\left(\mathcal{B} ; \mu^{-1} d X\right) \forall b \geq 2$ (cf. Proposition 9.10 of [20]).

(a) Here, our main ingredient is the following embedding theorem from section 8.8 of [20]. For $\gamma \in \mathbb{R} \backslash\{1\}$ we have

$$
H_{0}^{1}\left(\mathcal{B} ; d^{\gamma} d X\right) \hookrightarrow L^{2}\left(\mathcal{B} ; d^{\gamma-2} d X\right)
$$

Hence, $\psi \in \mathcal{V}$ implies

$$
\frac{\psi}{\mu} \in H_{0}^{1}\left(\mathcal{B} ; d^{\frac{b}{2}} d X\right) \hookrightarrow L^{2}\left(\mathcal{B} ; d^{\frac{b}{2}-2} d X\right)=L^{2}\left(\mathcal{B} ; \mu^{1-\frac{4}{b}} d X\right) .
$$

To analyze the term $\frac{\psi}{\mu} \nabla \Pi$ from $(2.29)$, we write

$$
\frac{\psi}{\mu} \nabla \Pi=\frac{\psi}{\mu^{1+\frac{2}{b}}} \nabla \Pi \mu^{\frac{2}{b}}=C \frac{\psi}{\mu^{1+\frac{2}{b}}} X e^{\frac{2}{b} X^{T} \kappa_{1}^{s} X} \in L^{2}(\mathcal{B} ; \mu d X)
$$

because of (2.31).

From (2.29) we hence obtain $\frac{\nabla \psi}{\mu} \in L^{2}(\mathcal{B} ; \mu d X)$ and $\psi \in H^{1}\left(\mathcal{B} ; \mu^{-1} d X\right)$ follows. For the reverse inclusion we use the embedding (2.30) with $\gamma=-\frac{b}{2}$, i.e.,

$$
\psi \in H_{0}^{1}\left(\mathcal{B} ; \mu^{-1} d X\right) \hookrightarrow L^{2}\left(\mathcal{B} ; \mu^{-1-\frac{4}{b}} d X\right)
$$

and proceed as in $(2.32)$. Now that $\mathcal{V}=H_{0}^{1}\left(\mathcal{B} ; \mu^{-1} d X\right)$ holds, Remark 9.17 of [20] yields the vanishing trace. 
(b) Since (2.30) holds for $\gamma=-1$ but not for $\gamma=1$, we obtain here only one of the inclusions between $\mathcal{V}$ and $H_{0}^{1}\left(\mathcal{B} ; \mu^{-1} d X\right)$. To analyze the trace in $\mathcal{V}$, take $\psi \in \mathcal{V} \cap$ $C^{\infty}(\overline{\mathcal{B}})$, which hence satisfies

$$
\int_{\mathcal{B}} \frac{\psi^{2}}{d(X)} d X<\infty
$$

Using spherical coordinates then shows $\psi(X)=0 \forall X \in \partial \mathcal{B}$ because of the nonintegrability at the boundary $\partial \mathcal{B}$ of the functions $d(X)^{-1}$. As a consequence of this boundary behavior we have

$$
\tilde{\mathcal{V}}:=\mathcal{V} \cap C^{\infty}(\overline{\mathcal{B}})=\left\{\psi \mid \frac{\psi}{\mu} \in C^{\infty}(\overline{\mathcal{B}})\right\} .
$$

To verify this, one can use, e.g., the Taylor formula for $\psi$ in local coordinates on $\partial \mathcal{B}$. Since $C^{\infty}(\overline{\mathcal{B}})$ is dense in $H^{1}(\mathcal{B} ; \mu d X)$ (cf. Th. 7.2 of $\left.[20]\right), \tilde{\mathcal{V}}$ is dense in $\mathcal{V}$. Hence, the (trivial) trace operator $\Gamma$ is already densely defined.

Following the decomposition of $\kappa$, we decompose $L$ as $L=L_{1}+L_{2}$.

$$
\begin{aligned}
& L_{1} \psi:=\frac{1}{2} \operatorname{div}\left(\left[\nabla \Pi-2 \kappa_{1} X\right] \psi+\nabla_{X} \psi\right)=\frac{1}{2} \operatorname{div}\left(\mu \nabla \frac{\psi}{\mu}\right), \\
& L_{2} \psi:=-\operatorname{div}\left(\kappa_{2} X \psi\right) .
\end{aligned}
$$

Next we define the associated quadratic forms

$$
\begin{aligned}
q_{1}(\psi, \varphi) & :=-\left\langle L_{1} \psi, \varphi\right\rangle=\frac{1}{2} \int_{\mathcal{B}} \nabla \frac{\psi}{\mu} \cdot \nabla \frac{\varphi}{\mu} \mu d X, \\
q_{2}(\psi, \varphi) & :=-\left\langle L_{2} \psi, \varphi\right\rangle=-\int_{\mathcal{B}} \frac{\psi}{\mu}\left(\nabla \frac{\varphi}{\mu}\right) \cdot \kappa_{2} X \mu d X, \\
q(\psi, \varphi) & :=q_{1}(\psi, \varphi)+q_{2}(\psi, \varphi),
\end{aligned}
$$

which are all bounded on $\mathcal{V}^{2}$. Note that $L_{1}$ (with form domain $\mathcal{V}$ ) is symmetric in $\mathcal{H}$, but $L_{2}$ is in general not anti-symmetric. From (2.33) it follows that the kernel of $L_{1}$ is spanned by $\mu$.

Using these quadratic forms we shall now give a weak reformulation of the steadystate problem (2.27)

Proposition 2.12. The weak formulation of (2.27) reads: Find $\phi \in \mu^{\perp}$ such that

$$
q(\phi, \varphi)=-q_{2}(\mu, \varphi), \quad \forall \varphi \in \mu^{\perp},
$$

with $\mu^{\perp}$ the closed subset of $\mathcal{V}$ defined by

$$
\mu^{\perp}:=\left\{\psi \in \mathcal{V} \mid \int_{\mathcal{B}} \psi d X=0\right\} .
$$

The weak solution of (2.27) is then $\psi:=\phi+\mu \in \mathcal{V}$.

Note that $\langle\psi, \mu\rangle=\int_{\mathcal{B}} \psi d X=0$ characterizes the orthogonal complement of $\mu$ in $\mathcal{H}$.

Proof. The problem to solve reads

$$
L \psi=0, \quad \text { with } \int_{\mathcal{B}} \psi d X=1, \psi \in \mathcal{V} .
$$


In order to cope with this normalization we proceed as in [3] and introduce $\phi:=\psi-\mu \in$ $\mu^{\perp}$. It satisfies

$$
L \phi=-L \mu=-L_{2} \mu, \quad \phi \in \mu^{\perp} .
$$

Taking the $\mathcal{H}$-inner product with $\varphi \in \mu^{\perp}$ yields the weak formulation (2.34).

Note that the possible boundary term disappears due to the zero trace (consider first test functions $\varphi \in \tilde{\mathcal{V}}$, which is dense in $\mathcal{V}$ ).

LEMMA 2.13.

(a) $L_{1}$ has a spectral gap $\lambda_{1}>0$.

(b) $L_{1}$ gives rise to the following Poincaré inequality:

$$
\left\|\frac{\psi}{\mu}\right\|_{L^{2}(\mathcal{B} ; \mu d X)}^{2} \leq \frac{1}{\lambda_{1}}\left\|\nabla \frac{\psi}{\mu}\right\|_{L^{2}(\mathcal{B} ; \mu d X)}^{2} \quad \forall \psi \in \mu^{\perp} .
$$

Proof.

(a) Since $\Pi$ is an "infinitely deep potential well" (i.e., $\Pi(X) \rightarrow \infty$ as $|X| \rightarrow \sqrt{b}$ ), $L_{1}$ has a positive spectral gap (for any choice of $\kappa_{1}$ !): This spectral gap $\lambda_{1}$ can be estimated with either of the following two arguments. First one could use the Bakry-Emerycondition $[6,4]$ for the potential $\Pi(X)-X^{T} \kappa_{1}^{s} X$, yielding

$$
\lambda_{1}>\lambda_{B E}:=\min _{X \in \mathcal{B}}\left[\lambda_{\min }\left(\operatorname{Hess}(\Pi(X))-2 \kappa_{1}^{s}\right)\right]=\lambda_{\min }\left(I-2 \kappa_{1}^{s}\right) .
$$

This yields a spectral gap if $\kappa_{1}^{s}<\frac{1}{2}$. An alternative estimate for the $\log$-Sobolev constant of $L_{1}$ and hence for its spectral gap is obtained by considering $-X^{T} \kappa_{1}^{s} X$ as an $L^{\infty}(\mathcal{B})$-perturbation of the potential $\Pi$. $\Pi$ is uniformly convex with

$$
\min _{X \in \mathcal{B}}\left[\lambda_{\min }(\operatorname{Hess} \Pi(X))\right]=1 .
$$

Using

$$
b \lambda_{\min }\left(\kappa_{1}^{s}\right) \leq X^{T} \kappa_{1}^{s} X \leq b \lambda_{\max }\left(\kappa_{1}^{s}\right), \quad X \in \mathcal{B},
$$

the Holley-Stroock perturbation argument $[16,4]$ yields for any $\kappa_{1}^{s}$

$$
\lambda_{1}>e^{-b\left[\lambda_{\max }\left(\kappa_{1}^{s}\right)-\lambda_{\min }\left(\kappa_{1}^{s}\right)\right]}>0 .
$$

We remark that neither of these estimates is sharp for the considered $\Pi$. The estimate (2.38), e.g., can be improved by optimizing on the decomposition of $\Pi$ into a uniformly convex function and a bounded perturbation.

(b) The spectral gap of $L_{1}$ gives rise to the Poincaré inequality (2.37) (e.g., put $g=\frac{\psi}{\mu}$ in section 3.3 of [4]).

Proposition 2.14. Let the spectral gap of $L_{1}$ and the matrix decomposition of $\kappa$ satisfy

$$
\sqrt{b}\left\|\kappa_{2}\right\|_{2}<\frac{\sqrt{\lambda_{1}}}{2}
$$

Then, the stationary Fokker-Planck equation (2.27), (2.26) admits a unique normalized weak solution $\psi_{\infty}=\phi+\mu \in \mathcal{V}$. 
Proof. We estimate with the Poincaré inequality for $\psi \in \mu^{\perp}$ :

$$
\left|q_{2}(\psi, \psi)\right| \leq \sqrt{b}\left\|\kappa_{2}\right\|_{2}\left\|\frac{\psi}{\mu}\right\|_{L^{2}(\mu d X)}\left\|\nabla \frac{\psi}{\mu}\right\|_{L^{2}(\mu d X)} \leq \sqrt{\frac{b}{\lambda_{1}}}\left\|\kappa_{2}\right\|_{2}\left\|\nabla \frac{\psi}{\mu}\right\|_{L^{2}(\mu d X)}^{2} .
$$

Hence, $q$ is coercive on $\mu^{\perp}$ :

$$
q(\psi, \psi) \geq\left(\frac{1}{2}-\sqrt{\frac{b}{\lambda_{1}}}\left\|\kappa_{2}\right\|_{2}\right)\left\|\nabla \frac{\psi}{\mu}\right\|_{L^{2}(\mu d X)}^{2} \geq\left(\frac{1}{2}-\sqrt{\frac{b}{\lambda_{1}}}\left\|\kappa_{2}\right\|_{2}\right) \frac{\lambda_{1}}{1+\lambda_{1}}\|\psi\|_{\mathcal{V}}^{2},
$$

and the assertion follows from the Lax-Milgram Lemma applied to (2.34).

We remark that the weak solution $\psi_{\infty}$ is independent of the decomposition $\kappa=\kappa_{1}+\kappa_{2}$ : Otherwise this would contradict the unique solvability of the weak formulation (2.34).

We shall now illustrate condition (2.39) for several typical decompositions of the shear matrix $\kappa$.

EXAMPLE 2.15. Choose $\kappa_{1}=\kappa^{\mathrm{s}}, \kappa_{2}=\kappa^{\mathrm{as}}$, and hence $\mu=C e^{-\Pi(X)+X^{T} \kappa^{\mathrm{s}} X}$. Then, condition (2.39) reads

$$
\sqrt{b}\left\|\kappa^{\mathrm{as}}\right\|_{2}<\frac{\sqrt{\lambda_{1}}}{2}
$$

To derive an alternative condition, $q_{2}$ can be rewritten here as

$$
\begin{aligned}
q_{2}(\psi, \psi) & =-\frac{1}{2} \int_{\mathcal{B}} \nabla^{T}\left(\frac{\psi}{\mu}\right)^{2} \kappa^{\mathrm{as}} X \mu d X=\frac{1}{2} \int_{\mathcal{B}}\left(\frac{\psi}{\mu}\right)^{2} \operatorname{div}\left(\kappa^{\mathrm{as}} X \mu\right) d X \\
& =\int_{\mathcal{B}}\left(\frac{\psi}{\mu}\right)^{2} X^{T} \kappa^{\mathrm{s}} \kappa^{\mathrm{as}} X \mu d X=-\frac{1}{4} \int_{\mathcal{B}}\left(\frac{\psi}{\mu}\right)^{2} X^{T}\left[\kappa, \kappa^{T}\right] X \mu d X .
\end{aligned}
$$

Estimating as in (2.40) yields the following alternative condition for Proposition 2.14 to hold:

$$
b\left\|\left[\kappa, \kappa^{T}\right]\right\|_{2}<2 \lambda_{1}
$$

EXAmple 2.16. Choose $\kappa_{1}=\kappa^{\text {as }}, \kappa_{2}=\kappa^{\mathrm{s}}$, and hence $\mu=C e^{-\Pi(X)}, \lambda_{1}>\lambda_{B E}=1$. Then, condition $(2.39)$ reads

$$
\sqrt{b}\left\|\kappa^{\mathrm{s}}\right\|_{2}<\frac{\sqrt{\lambda_{1}}}{2}
$$

EXAMPLE 2.17. With section 2.3 in mind, another obvious option is to choose $\kappa_{1}$ as the closest normal matrix to $\kappa$ [30], and $\kappa_{2}$ as the non-normal remainder. We refer to [22] for estimates between this non-normal remainder and the commutator $\left[\kappa, \kappa^{T}\right]$.

Next we turn to the large-time convergence of the Fokker-Planck solution $\psi(t)$ towards the steady state $\psi_{\infty}$.

ThEOREM 2.18. Let $\psi_{0} \in \mathcal{H}:=L^{2}\left(\mathcal{B} ; \mu^{-1} d X\right)$. Then, the Fokker-Planck equation (2.25), (2.26) has a unique weak solution $\psi \in L^{2}((0, T), \mathcal{V}) \cap H^{1}\left((0, T), \mathcal{V}^{\prime}\right) \cap C([0, T], \mathcal{H})$ 
for any $T>0$. Moreover, $\int \psi_{0} d X=\int \psi(t) d X, \forall t \geq 0$. For $\psi_{0}$ normalized and under the assumptions of Proposition 2.14 it satisfies

$$
\left\|\psi(t)-\psi_{\infty}\right\|_{\mathcal{H}} \leq e^{-\lambda_{1}\left(\frac{1}{2}-\sqrt{\frac{b}{\lambda_{1}}}\left\|\kappa_{2}\right\|_{2}\right) t}\left\|\psi_{0}-\psi_{\infty}\right\|_{\mathcal{H}}, \quad t>0,
$$

and analogously under Assumption (2.43). Moreover, $\psi_{\infty}(X) \geq 0$.

Proof. Using $q(\psi, \varphi)=-\langle L \psi, \varphi\rangle$ we see that $L \in \mathcal{L}\left(\mathcal{V}, \mathcal{V}^{\prime}\right)$. Since $q$ satisfies on $\mathcal{V}$ the following modified coercivity estimate (use (2.33), (2.40) and Young's inequality):

$$
q(\psi, \psi) \geq\left(\frac{1}{2}-\varepsilon\right)\|\psi\|_{\mathcal{V}}^{2}-C(\varepsilon)\|\psi\|_{\mathcal{H}}^{2},
$$

standard parabolic theory (cf. section 11.1 of [29], e.g.) yields the first assertion. Moreover, this solution satisfies a.e. in $(0, T)$ :

$$
\frac{d}{d t}\|\psi(t)\|_{\mathcal{H}}^{2}=2 \mathcal{V}^{\prime}\left\langle\psi^{\prime}(t), \psi(t)\right\rangle_{\mathcal{V}}=-2 q(\psi(t), \psi(t)) .
$$

For normalized $\psi_{0}$ we have $\psi(t)-\psi_{\infty} \in \mu^{\perp}$, a.e. in $(0, \infty)$ by using $\mu$ as a test function in the weak formulation of the equation. Hence, (2.41) and the Poincaré inequality yield

$$
\frac{d}{d t}\left\|\psi(t)-\psi_{\infty}\right\|_{\mathcal{H}}^{2} \leq-2 \lambda_{1}\left(\frac{1}{2}-\sqrt{\frac{b}{\lambda_{1}}}\left\|\kappa_{2}\right\|_{2}\right)\left\|\psi(t)-\psi_{\infty}\right\|_{\mathcal{H}}^{2}, \quad \text { a.e. in }(0, T),
$$

and the exponential convergence follows.

The fact that $\psi(t)-\psi_{\infty} \in \mu^{\perp}$ and $\psi \in C([0, T], \mathcal{H})$ imply the conservation of mass. To prove the non-negativity of $\psi_{\infty}$ we choose an arbitrary non-negative, normalized $\psi_{0} \in \mathcal{H} . \psi(t, X) \geq 0$ then implies $\psi_{\infty}(X) \geq 0$.

We remark that the existence part of the above theorem (in an equivalent norm) was already sketched in Appendix B of [19]. We only included it for the sake of completeness.

Following the procedure of Remark 13 in [19], one can deduce that the weak solutions from Proposition 2.14 and Theorem 2.18 then satisfy the no-flux boundary condition a.e. in $t$ in the following sense:

$$
\int_{\partial \mathcal{B}}\left(\frac{1}{2} \nabla \frac{\psi(t)}{\mu}-\kappa_{2} X \frac{\psi(t)}{\mu}\right) \cdot n \chi \mu d S=0 \quad \forall \chi \in H^{1}(\mathcal{B} ; \mu d X),
$$

with $n$ being the unit outward normal vector on $\partial \mathcal{B}$. By Lemma 2.11 this simplifies to

$$
\int_{\partial \mathcal{B}} \nabla \frac{\psi(t)}{\mu} \cdot n \chi \mu d S=0 \quad \forall \chi \in H^{1}(\mathcal{B} ; \mu d X) .
$$

One strategy to extend the above large time convergence to the logarithmic relative entropy (w.r.t. the stationary state $\psi_{\infty}$ ) would be to apply a Holley-Stroock perturbation argument. To this end we would have to show that there exist constants $C_{1}, C_{2}$ such that

$$
0<C_{1} \leq \psi_{\infty} / \mu \leq C_{2},
$$

as done in $[19$, Proposition 10, Lemma 6$]$ in their case. However, we do not know how to show these bounds in the present case. 


\section{Coupled model: large time behavior}

In this section, we derive exponential convergence results towards homogeneous stationary flow solutions of the coupled problem

$$
\begin{aligned}
\frac{\partial u}{\partial t}+\left(u \cdot \nabla_{x}\right) u & =\Delta_{x} u-\nabla_{x} p+\operatorname{div}_{x} \int_{\mathrm{IR}^{d}}\left(X \otimes \nabla_{X} \Pi(X)\right) \psi d X \\
\operatorname{div}_{x} u & =0, \\
\frac{\partial \psi}{\partial t}+u \cdot \nabla_{x} \psi & =-\operatorname{div}_{X}\left(\left[\left(\nabla_{x} \otimes u\right)^{T} \cdot X-\frac{1}{2} \nabla_{X} \Pi(X)\right] \psi\right)+\frac{1}{2} \Delta_{X} \psi .
\end{aligned}
$$

More precisely, this problem is posed in a bounded spatial domain $x \in \Omega$ and in the configuration space $B \subset \mathbb{R}^{d}$ with $B=\mathbb{R}^{d}$ in the Hookean case or $B=\mathcal{B}(0, \sqrt{b})$, the Euclidean ball centered at 0 with radius $\sqrt{b}$, in the FENE model. These equations have to be complemented by boundary conditions in such a way that $u_{\infty}=\kappa x$, with $\kappa$ any traceless real matrix, and $\psi_{\infty}=\psi_{\infty}(X)$ form a stationary solution. Here, $\psi_{\infty}$ is given by the stationary solution obtained in section 2 either in the Hookean or in the FENE case. These solutions were called homogeneous stationary flows in $[19$, section 3.3]. These boundary conditions amount to $u=u_{\infty}$ on $\partial \Omega$ and $\psi=0$ on $\partial \mathcal{B}(0, \sqrt{b})$ in the FENE case or decay at infinity of the solution $\psi$ in the Hookean case. The latter condition is usually imposed by the class of solutions we work with. We will refer to these boundary conditions as non-homogeneous stationary Dirichlet boundary conditions as in [19].

In this section we will concentrate on the Hookean case, in particular on the long time asymptotics of smooth solutions to (3.1), where $u$ satisfies the above boundary conditions and $\psi$ has a fast decay at infinity. Such solutions in the Hookean case are known to exist for small initial data when $\kappa=0$ [23] with a smallness condition in some suitably chosen high-order Sobolev space. Similar results are quite likely to hold for the above non-homogeneous boundary conditions in a suitable neighborhood of the steady state $\left(u_{\infty}, \psi_{\infty}\right)$. Let us remark that in these "close to equilibrium" results, one obtains (see [23]) that the deformation matrix $\nabla_{x} \otimes u^{s}:=\frac{1}{2}\left(\nabla_{x} \otimes u+\left(\nabla_{x} \otimes u\right)^{T}\right)$ is globally bounded, i.e., that

$$
D:=\sup _{0<t<\infty}\left\|\nabla_{x} \otimes u^{s}(t, \cdot)\right\|_{L^{\infty}(\Omega)}<\infty .
$$

We will make this bounded deformation matrix assumption in the rest of this section. In far-from-equilibrium situations, however, the existence of such global solutions remains an open problem.

The objective of this section is to improve over the results obtained in [19] in the coupled case. More precisely, we will show an exponential rate of convergence in the Hookean case under some smallness assumption, a result stated as an open problem in $[19$, section 3.3$]$.

One of the technical ingredients is the following generalization of the CsiszárKullback inequality obtained by a similar proof to the standard Csiszár-Kullback inequality $[13,21]$. It can also be derived from the Csiszár-Kullback inequality and standard moment interpolation.

LEMMA 3.1. Let $\psi, \varphi \in L_{+}^{1}\left(\mathbb{R}^{d}\right)$ with unit mass such that $\varphi>0$ with bounded fourth moments, i.e., $|X|^{4}(\psi+\varphi) \in L^{1}\left(\mathbb{R}^{d}\right)$. Then, the following inequality holds:

$$
\left\||X|^{2}(\psi-\varphi)\right\|_{L^{1}\left(\mathbb{R}^{d}\right)}^{2} \leq 2 e(\psi \mid \varphi) \max \left(\int_{\mathbb{R}^{d}}|X|^{4} \psi d X, \int_{\mathbb{R}^{d}}|X|^{4} \varphi d X\right) .
$$


Proof. By a Taylor expansion at order two of $s \log s$ at $s=1$ and the normalization of mass, we can write the relative entropy for $\psi$ and $\varphi$ as

$$
e(\psi \mid \varphi)=\int_{\mathbb{R}^{d}} \frac{\psi}{\varphi} \ln \frac{\psi}{\varphi} \varphi d X=\frac{1}{2} \int_{\mathcal{A}} \frac{1}{\xi}(\psi-\varphi)^{2} d X
$$

where $\mathcal{A}:=\left\{X \in \mathbb{R}^{d}: \psi(X) \neq \varphi(X)\right\}$ and $\xi(X)$ lies between $\psi(X)$ and $\varphi(X)$, i.e., $0 \leq$ $\min (\psi, \varphi)<\xi<\max (\psi, \varphi)$ in $\mathcal{A}$, and thus $\xi>0$ in $\mathcal{A}$. By Hölder's inequality and since $\mathcal{A}$ is measurable, we obtain

$$
\int_{\mathcal{A}}|X|^{2}|\psi-\varphi| d X \leq\left(\int_{\mathcal{A}} \frac{1}{\xi}(\psi-\varphi)^{2} d X\right)^{1 / 2}\left(\int_{\mathcal{A}}|X|^{4} \xi d X\right)^{1 / 2},
$$

from which the stated inequality (3.3) is obtained.

Now, let us show that the assumption of a bounded deformation matrix (3.2), implies the boundedness in space and time of all moments of the distribution function $\psi$ and in particular of the stress tensor in the Hookean case, if they are initially bounded. In order to verify this, we multiply the equation for $\psi$ by $|X|^{2 n}$, with $n \in \mathbb{I N}$ and integrate to obtain

$$
\begin{aligned}
\frac{\partial m_{2 n}(\psi)}{\partial t}+u \cdot \nabla_{x} m_{2 n}(\psi)= & 2 n \int_{\mathbb{I R}^{d}} X^{T}\left(\nabla_{x} \otimes u^{s}\right) X|X|^{2 n-2} \psi d X \\
& -n m_{2 n}(\psi)+n(2 n-2+d) m_{2 n-2}(\psi),
\end{aligned}
$$

where

$$
m_{2 n}(\psi)(t, x):=\int_{\mathbb{R}^{d}}|X|^{2 n} \psi(t, x, X) d X .
$$

Using the assumption (3.2), we obtain

$$
\frac{\partial m_{2 n}(\psi)}{\partial t}+u \cdot \nabla_{x} m_{2 n}(\psi) \leq n(2 D-1) m_{2 n}(\psi)+n(2 n-2+d) m_{2 n-2}(\psi)
$$

for all $n \in \mathbb{I N}$. Assuming that the inhomogeneity is small enough, i.e., $D<\frac{1}{2}$, then we obtain

$$
\frac{d}{d t} M_{2 n}(\psi) \leq-A_{n} M_{2 n}(\psi)+B_{n} M_{2 n-2}(\psi)
$$

where $M_{2 n}(\psi)(t, x)=m_{2 n}(\psi)\left(t, \Phi_{t}(x)\right)$ with $\Phi_{t}$ the flow map associated to the velocity field $u$, i.e.,

$$
\left\{\begin{aligned}
\frac{d \Phi_{t}(x)}{d t} & =u\left(t, \Phi_{t}(x)\right) & & t \geq 0, \\
\Phi_{0}(x) & =x & & x \in \mathbb{R}^{d} .
\end{aligned}\right.
$$

Now, a simple induction argument starting at $n=1$ for which $M_{0}(\psi)=1$, implies that

$$
M_{2 n}:=\max _{0 \leq t<\infty}\left\|\int_{\mathbb{R}^{d}}|X|^{2 n} \psi(t, x, X) d X\right\|_{L^{\infty}(\Omega)}<\infty
$$


if initially $m_{2 n}\left(\psi_{0}\right) \in L^{\infty}(\Omega)$, i.e.,

$$
\left\|\int_{\mathbb{R}^{d}}|X|^{2 n} \psi_{0}(x, X) d X\right\|_{L^{\infty}(\Omega)}<\infty .
$$

Actually, the assumption $D<\frac{1}{2}$ involving (3.2) could be slightly weakened to

$$
\sup _{0<t<\infty} \sup _{x \in \Omega} \lambda_{\max }\left(\nabla_{x} \otimes u^{s}(t, x)\right)<\frac{1}{2},
$$

which is a closely related analogue of $2 \kappa-I$ having to be a stable matrix (cf. the condition in Proposition 2.1).

Now, with these estimates together with the entropy-entropy dissipation procedure applied in [19, section 3.3.1], we can deduce an exponential convergence result towards homogeneous stationary flows in the Hookean case with small enough initial data. Now we define the total relative entropy of a solution of the coupled model $(u, \psi)$ to the homogeneous stationary flow $\left(u_{\infty}, \psi_{\infty}\right)$ as:

$$
E(t)=\frac{1}{2} \int_{\Omega}|\bar{u}(t)|^{2} d x+\int_{\Omega} \int_{\mathbb{R}^{d}} \psi(t) \ln \left(\frac{\psi(t)}{\psi_{\infty}}\right) d X d x,
$$

with $\bar{u}:=u-u_{\infty}$. Then one has the following formula for its evolution, see [19, Appendix A]:

$$
\begin{aligned}
\frac{d E}{d t}+\int_{\Omega}\left|\nabla_{x} \otimes \bar{u}\right|^{2} d x & +\frac{1}{2} \int_{\Omega} \int_{\mathbb{R}^{d}} \psi\left|\nabla_{X} \ln \left(\frac{\psi}{\psi_{\infty}}\right)\right|^{2} d X d x \\
& =-\int_{\Omega} \bar{u}^{T} \kappa \bar{u} d x-\int_{\Omega} \int_{\mathbb{R}^{d}} \nabla_{X} \ln \left(\frac{\psi_{\infty}}{e^{-\Pi(X)}}\right)^{T}\left(\nabla_{x} \otimes \bar{u}\right) X \bar{\psi} d X d x
\end{aligned}
$$

with $\bar{\psi}:=\psi-\psi_{\infty}, \Pi(X)=\frac{1}{2}|X|^{2}$.

THEOREM 3.2. Let us consider the homogeneous stationary flow $\left(u_{\infty}=\kappa x, \psi_{\infty}\right)$ with the traceless matrix $\kappa$ satisfying $\Re \lambda_{j}(\kappa)<\frac{1}{2}, j=1, \ldots, d$ and $\psi_{\infty}$ given by (2.11). Let $(u, \psi)$ be a given smooth, fast-decaying at infinity solution to the system (3.1) with non-homogeneous Dirichlet boundary conditions such that the deformation matrix is uniformly bounded (in the sense of (3.2)). Then, the solution converges exponentially towards $\left(u_{\infty}, \psi_{\infty}\right)$, provided a "smallness" condition holds for the solution and $\kappa^{s}$ as specified in (3.8). More precisely, $u$ converges exponentially to $u_{\infty}$ in $L^{2}(\Omega)$ and the total relative entropy of $\psi$ w.r.t. $\psi_{\infty}$ converges exponentially to 0.

Proof. We proceed as in [19, Theorem 1] for the FENE case. The potential $\frac{1}{2} X^{T} \Sigma^{-1} X=C-\ln \psi_{\infty}$ (cf. (2.11)) satisfies the Bakry-Emery condition with constant $\lambda=\lambda_{\min }\left(\Sigma^{-1}\right)$. Using the resulting Logarithmic Sobolev inequality for the measure $\psi_{\infty} d X$ in (3.7) we obtain

$$
\begin{aligned}
\frac{d E}{d t} & +\int_{\Omega}\left|\nabla_{x} \otimes \bar{u}\right|^{2} d x+\lambda \int_{\Omega} \int_{\mathbb{R}^{d}} \psi \ln \left(\frac{\psi}{\psi_{\infty}}\right) d X d x \\
& \leq\left|\kappa^{s}\right| \int_{\Omega}|\bar{u}|^{2} d x+\int_{\Omega}\left|\nabla_{x} \otimes \bar{u}\right| \int_{\mathbb{R}^{d}}\left|\nabla_{X} \ln \left(\frac{\psi_{\infty}}{e^{-\Pi(X)}}\right)\right||X||\bar{\psi}| d X d x .
\end{aligned}
$$

Applying Young's inequality with $\epsilon<1$ to be chosen, using the Poincaré inequality 
on $\Omega$, and the explicit formula of $\psi_{\infty}$, we obtain

$$
\begin{aligned}
& \frac{d E}{d t}+\frac{1-\epsilon}{C_{P}^{2}} \int_{\Omega}|\bar{u}|^{2} d x+\lambda \int_{\Omega} \int_{\mathbb{R}^{d}} \psi \ln \left(\frac{\psi}{\psi_{\infty}}\right) d X d x \\
& \leq\left|\kappa^{s}\right| \int_{\Omega}|\bar{u}|^{2} d x+\frac{\left|I-\Sigma^{-1}\right|^{2}}{4 \epsilon} \int_{\Omega}\left(\int_{\mathbb{R}^{d}}|X|^{2}|\bar{\psi}| d X\right)^{2} d x .
\end{aligned}
$$

Now, we apply the inequality (3.3) proved in Lemma 3.1 to obtain

$$
\begin{aligned}
\frac{d E}{d t}+ & \frac{1-\epsilon}{C_{P}^{2}} \int_{\Omega}|\bar{u}|^{2} d x+\lambda \int_{\Omega} \int_{\mathbb{R}^{d}} \psi \ln \left(\frac{\psi}{\psi_{\infty}}\right) d X d x \\
& \leq\left|\kappa^{s}\right| \int_{\Omega}|\bar{u}|^{2} d x+\frac{\left|I-\Sigma^{-1}\right|^{2}}{2 \epsilon} \max \left(M_{4}, M_{4}^{\infty}\right) \int_{\Omega} \int_{\mathbb{R}^{d}} \psi \ln \left(\frac{\psi}{\psi_{\infty}}\right) d X d x
\end{aligned}
$$

where $M_{4}$ is given by (3.5) and $M_{4}^{\infty}$ is the fourth moment of $\psi_{\infty}$. From here it is clear that, if $\epsilon$ can be chosen such that

$$
\epsilon<1-C_{P}^{2}\left|\kappa^{s}\right| \text { and } \epsilon>\frac{\left|I-\Sigma^{-1}\right|^{2}}{2 \lambda} \max \left(M_{4}, M_{4}^{\infty}\right),
$$

or equivalently if

$$
C_{P}^{2}\left|\kappa^{s}\right|+\frac{\left|I-\Sigma^{-1}\right|^{2}}{2 \lambda} \max \left(M_{4}, M_{4}^{\infty}\right)<1,
$$

then exponential convergence holds.

The previous result is the main new addition to the results in [19] concerning the long time asymptotics of the coupled problem. Concerning the FENE case, we have shown in section 2.4 the existence of the stationary state $\psi_{\infty}$ in several new situation not covered in [19]. Those stationary states $\psi_{\infty}$ that eventually verify the bounds (2.45), give rise to a Logarithmic Sobolev inequality via a Holley-Stroock perturbation argument. In these cases we can derive the corresponding long time asymptotics result of the coupled problem just by repeating the proof of $[19$, Theorem 1] or [19, Proposition 9].

Acknowledgement. AA was partially supported by the ESF in the project "Global and geometrical aspects of nonlinear partial differential equations" and the Wissenschaftskolleg Differentialgleichungen of the FWF. JAC acknowledges the support from DGI-MCI (Spain) project MTM2008-06349-C03-03 and 2009-SGR-345 from AGAUR-Generalitat de Catalunya. AA and JAC acknowledge partial support of the Acc. Integ. program HU2006-0025. JAC and CM acknowledge partial support of the Acc. Integ. program HI2006-0111. This research was (partially) completed while the first two authors were visiting the Institute for Mathematical Sciences, National University of Singapore in 2009.

\section{REFERENCES}

[1] A. Arnold and E. Carlen, A generalized Bakry-Emery condition for non-symmetric diffusions, in EQUADIFF 99 - Proceedings of the Internat. Conference on Differential Equations, Berlin 1999, B. Fiedler, K. Gröger, J. Sprekels (Eds.); World Scientific Publishing, 732$734,2000$.

[2] A. Arnold, E. Carlen and Q. Ju, Large-time behavior of non-symmetric Fokker-Planck type equations, Commun. Stochastic Anal., 2(1), 153-175, 2008. 
[3] A. Arnold, I.M. Gamba, M.P. Gualdani and C. Sparber, The Wigner-Fokker-Planck equation: stationary states and large time behavior, preprint, 2008.

[4] A. Arnold, P. Markowich, G. Toscani and A. Unterreiter, On convex Sobolev inequalities and the rate of convergence to equilibrium for Fokker-Plack type equations, Commun. PDE, $26,43-100,2001$.

[5] A. Arnold and A. Unterreiter, Entropy decay of discretized Fokker-Planck equations I - temporal semi-discretization, Comput. Math. Appl., 46(10-11), 1683-1690, 2003.

[6] D. Bakry and M. Emery, Hypercontractivité de semi-groupes de diffusion, C.R. Acad. Sci. Paris Sér., I, 15, 299-305, 1984.

[7] D. Bakry and M. Emery, Diffusions hypercontractivities, Sém. Prob. XIX, Lecture Notes in Math. Springer, 1123, 177-206, 1985.

[8] R.H. Bartels and G.W. Stewart, Algorithm 432: solution of the matrix equation $A X+X B=C$, Commun. ACM, 15, 820-826, 1972.

[9] R.B. Bird, R.C. Armstrong and O. Hassager, Dynamics of Polymeric Liquids, Wiley Interscience, 1, 1987.

[10] R.B. Bird, C.F. Curtiss, R.C. Armstrong and O. Hassager, Dynamics of Polymeric Liquids, Wiley Interscience, 2, 1987.

[11] M. Brookes, The Matrix Reference Manual, (online), 2005. http://www.ee.ic.ac.uk/hp/staff/dmb/matrix/intro.html

[12] L. Chupin, The FENE model for viscoelastic thin film flows, Methods and Appl. of Analysis, 16(1), 217-262, 2009

[13] I. Csiszár, Information-type measures of difference of probability distributions and indirect observations, Stud. Sci. Math. Hung., 2, 299-318, 1967.

[14] M. Doi and S.F. Edwards, The Theory of Polymer Dynamics, International Series of Monographs on Physics, Oxford University Press, Oxford, 1988.

[15] Q. Du, C. Liu and P. Yu, FENE dumbbell model and its several linear and nonlinear closure approximations, Multiscale Model. Simul., 4, 709-731, 2005.

[16] R. Holley and D. Stroock, Logarithmic Sobolev inequalities and stochastic Ising models, J. Stat. Phys., 46(5-6), 1159-1194, 1987.

[17] R.A. Horn and C.R. Johnson, Topics in Matrix Analysis, Cambridge University Press, Cambridge, 1991.

[18] Y. Hyon, J.A. Carrillo, Q. Du and C. Liu, A macroscopic closure approximation to the micromacro FENE models with maximum entropy principle for polymeric materials, Kinetic and Related Models, 2, 171-184, 2008.

[19] B. Jourdain, C. Le Bris, T. Lelièvre and F. Otto, Long-time asymptotics of a multiscale model for polymeric fluid flows, Arch. Rational Mech. Anal., 181, 97-148, 2006.

[20] A. Kufner, Weighted Sobolev Spaces, Teubner, Leipzig, 1980.

[21] S. Kullback and R.A. Leibler, On information and sufficiency, Annals of Mathematical Statistics, 22, 79-86, 1951.

[22] L. László, An attainable lower bound for the best normal approximation, SIAM J. Matrix Analysis and Applications, 15, 1035-1043, 1994.

[23] F. Lin, C. Liu and P. Zhang, On a micro-macro model for polymeric fluids near equilibrium, Commun. Pure Appl. Math., 60(6), 838-866, 2007.

[24] C. Liu and H. Liu, Boundary conditions for the microscopic FENE models, SIAM J. Appl. Math., 68(5), 1304-1315, 2008.

[25] H. Liu and J. Shin, Global well-posedness for the microscopic FENE model with a sharp boundary condition, arXiv:0905.1142 (preprint posted 06.06.2009).

[26] P.A. Markowich and C. Villani, On the trend to equilibrium for the Fokker-Plack equation: an interplay between physics and functional analysis, Mat. Contemp., 19, 1-29, 2000.

[27] N. Masmoudi, Well posedness for the FENE dumbbell model of polymeric flows, CPAM, 61(12), 1685-1714, 2008.

[28] R. Owens and T. Phillips, Computational Rheology, Imperial College Press, London, 2002.

[29] M. Renardy and R.C. Rogers, An Introduction to Partial Differential Equations, 2nd Edition, Springer, New York, 2004.

[30] A. Ruhe, Closest normal matrix finally found! BIT, 27, 585-598, 1987.

[31] J. Snyders and M. Zakai, On nonnegative solutions of the equation $A D+D A^{\prime}=-C$, SIAM J. Appl. Math., 18, 704-714, 1970.

[32] H. Triebel, Interpolation Theory, Function Spaces, Differential Operators, Barth Verlag, Heidelberg-Leipzig, 1995.

[33] H. Zhang and P. Zhang, Local existence for the FENE-dumbbell model of polymeric fluids, Arch. Ration. Mech. Anal., 181, 373-400, 2006. 